\title{
How to Embed a Path onto Two Sets of Points
}

\author{
Emilio Di Giacomo, Giuseppe Liotta, and Francesco Trotta \\ Dipartimento di Ingegneria Elettronica e dell'Informazione, \\ Università degli Studi di Perugia \\ \{digiacomo, liotta, francesco.trotta\}@diei.unipg.it
}

\begin{abstract}
Let $R$ and $B$ be two sets of points such that the points of $R$ are colored red and the points of $B$ are colored blue. Let $P$ be a path such that $|R|$ vertices of $P$ are red and $|B|$ vertices of $P$ are blue. We study the problem of computing a crossing-free drawing of $P$ such that each blue vertex is represented as a point of $B$ and each red vertex of $P$ is represented as a point of $R$. We show that such a drawing can always be realized by using at most one bend per edge.
\end{abstract}

\section{Introduction}

Let $G$ be a planar graph such that each vertex of $G$ is colored with either the red or the blue color. Let $R$ and $B$ be two distinct sets of red and blue points in the plane, respectively, such that $|R|$ equals the number of red vertices of $G$ and $|B|$ equals the number of blue vertices of $G$. A bichromatic point-set embedding of $G$ onto $R \cup B$ is a crossing-free drawing such that those vertices that are blue in $G$ are mapped to points of $B$ and those vertices that are red in $G$ are mapped to points of $R$. The mapping of each blue/red vertex of $G$ to a corresponding blue/red point of $R \cup B$ is not part of the input.

The problem of computing bichromatic point-set embeddings for different subclasses of planar graphs has attracted considerable interest during the last fifteen years. We briefly recall here only some of the most relevant results concerning the case that $G$ is a simple path, since this is the main subject of this short paper. For an exhaustive survey see [5]. In what follows we shall denote with $S$ the set $R \cup B$ and implicitly assume that the red (blue) points of $S$ are always as many as the red (blue) vertices of the bi-colored input path $P$.

Akiyama and Urrutia [2] exhibit a set $S$ of sixteen points in convex position on which a proper 2-colored path $P$ does not admit a straight-line bichromatic point-set embedding, and present an $O\left(n^{2}\right)$-time algorithm to test whether a proper 2-colored path has a straight-line bichromatic point-set embedding on a given set of points. Abellanas et al. [1] also study straight-line point-set embeddings for a path $P$ with a proper 2-coloring. They show that if either the convex hull of $S$ consists of all red points and no blue points or $S$ is a linearly separable bipartition (i.e. there exists a line that separates all blue points from the red ones), then $P$ has a straight-line point-set embedding onto $S$. Finally, a recent paper by Kaneko, Kano, and Suzuki [4] provides a complete characterization of those paths with a proper 2-coloring that admit a straight-line bichromatic 
point-set embedding onto any set of points $S$ in general position: If $P$ has at most twelve vertices or if it has exactly fourteen vertices, then $P$ always admits a straight-line bichromatic point-set embedding onto $S$; for all other cases, there exist configurations of $S$ for which $P$ does not admit a straight-line bichromatic point-set embedding.

Motivated by the result of Kaneko, Kano, and Suzuki [4, we study the problem of constructing a bichromatic point-set embedding of a 2-colored path by removing the restriction that no three points of $S$ are collinear and by not assuming that the given 2-coloring is proper. We observe that allowing collinearities naturally leads to bichromatic point set embeddings whose edges can contain bends. The main contribution of this paper is the following theorem.

Theorem 1. Let $P$ be a simple path such that each vertex of $P$ is colored with either the red or the blue color. Let $R$ and $B$ be two distinct sets of points in the plane such that $|R|$ equals the number of red vertices of $P$ and $|B|$ equals the number of blue vertices of $P$. Then $P$ admits a bichromatic point-set embedding onto $R \cup B$ with at most one bend per edge.

The proof of Theorem 1 is based on showing that a 2-colored path admits a bichromatic point-set embedding onto any given set $S$ if and only if it has a suitably defined 2-page bichromatic book embedding (see Section 2).

\section{Preliminaries}

Let $G=(V, E)$ be a planar graph. A 2-coloring of $G$ is a partition of $V$ into 2 disjoint sets $V_{b}$ and $V_{r}$. We call blue vertices the vertices of $V_{b}$ and red vertices the vertices of $V_{r}$. A 2-coloring is proper if for every edge $(u, v) \in E$ we have $u \in V_{b}$ and $v \in V_{r}$. Given a vertex $v$ we denote by $c(v)$ the color of $v$. If a graph $G$ has a 2 -coloring we say that it is 2 -colored, if the 2-coloring is proper we say that $G$ is properly 2 -colored.

Let $G$ be a planar 2-colored graph and let $S=B \cup R$ be a set of points in the plane, such that $|B|=\left|V_{b}\right|$ and $|R|=\left|V_{r}\right|$. We call blue points the points of $B$ and red points the points of $R$. A point-set embedding onto $S$ of $G$ is a planar drawing $\Gamma$ such that the vertices of $G$ are drawn in $\Gamma$ on the points of $S$, and each edge of $G$ is drawn as a polyline in $\Gamma$ (Kaufmann and Wiese [6] show that any planar graph admits a points-set embedding). $G$ has a bichromatic point-set embedding onto $S$ if $G$ has a point-set embedding onto $S$ such that every blue vertex is drawn on a blue point, and every red vertex is drawn on a red point. A planar 2-colored graph $G$ is bichromatic point-set embeddable if for any set of points, $S=R \cup B$ such that $|B|=\left|V_{b}\right|$ and $|R|=\left|V_{r}\right|, G$ has a bichromatic point-set embedding onto $S$.

Let $G$ be a planar graph. An $h$-page book embedding of $G$ consists of a linear ordering $\lambda$ of the vertices of $G$ and a partition of the edges of $G$ into $h$ disjoint sets, called pages, such that there are no two edges $(u, v)$ and $(w, z)$ in the same page with $u<w<v<z$ in $\lambda$. A different but equivalent definition of an $h$-page book embedding is the following. An $h$-page book embedding of $G$ is a drawing 
of $G$ such that all the vertices of $G$ are drawn as points of a straight line $l$ called spine, each edge is drawn on one of $h$ half-planes, called pages, having $l$ as a common boundary, and no two edges in the same page cross. According to this second definition, a book embedding is a drawing rather than a combinatorial object. In the following we shall always refer to this "geometric" definition rather than to the "combinatorial" one. In the special case when $h=2$ we have that a 2-page book embedding of $G$ is a planar drawing such that all the vertices are drawn as points of a straight line $l$, and each edge is drawn on one of the two half-planes defined by $l$.

A red-blue sequence $\sigma$ is a sequence of points along a straight line $l$ such that each point $p \in \sigma$ is either red or blue. Given a point $p$ of $\sigma$, we denote by $c(p)$ the color of $p$. Let $n_{r}$ and $n_{b}$ be the number of red and blue points in a redblue sequence $\sigma$, respectively, and let $G$ be a planar 2-colored graph such that $\left|V_{b}\right|=n_{b}$ and $\left|V_{r}\right|=n_{r}$. An $h$-page book embedding of $G$ consistent with $\sigma$ is an $h$-page book embedding of $G$ such that each vertex $v$ of $G$ is represented by a point $p$ of $\sigma$ and $c(v)=c(p)$. Notice that the exact position of the points of $\sigma$ on the line $l$ is not relevant for the existence of the book embedding, and only their relative order is important. A planar 2-colored graph $G$ is h-page bichromatic book embeddable if, for any red-blue sequence $\sigma$ with $\left|V_{b}\right|=n_{b}$ and $\left|V_{r}\right|=n_{r}$, $G$ has an $h$-page book embedding consistent with $\sigma$. Let $\gamma$ be an $h$-page book embedding of $G$, and let $v$ be a vertex of $G$. We say that $v$ is accessible from a page $\pi$ if there is no edge $(u, w)$ in $\pi$ such that $u<v<w$ in the linear ordering of $\gamma$. Analogously we say that a point $p \in \sigma$ is accessible from a page $\pi$ if there is no edge $(u, w)$ in $\pi$ such that $u<p<w$ in the linear ordering of $\gamma$. Two vertices/points accessible from a common page can be connected by an edge without creating any crossings.

In [3] it has been proved that there is a strong connection between point-set embeddability and book embeddability. More precisely, the following lemma is an immediate consequence of 3 .

Lemma 1. [3] Let $G$ be a planar graph. G admits a 2-page book embedding if and only if $G$ admits a point-set embedding with at most 1 bend per edge on any set of points.

The following theorem shows that the result can be extended to the case of bichromatic point-set embedding and bichromatic book embedding. The proof is omitted for reasons of space.

Theorem 2. Let $G$ be a planar 2-colored graph. Then $G$ is bichromatic point-set embeddable with at most 1 bend per edge if and only if it is 2-page bichromatic book embeddable.

\section{Bichromatic Point-Set Embedding of Paths}

In this section we prove Theorem 1 and apply it to the bichromatic point-set embeddability of cycles. Based on Theorem 2 , it suffices to prove the following. 
Theorem 3. Let $P$ be a 2-colored path, and let $\sigma$ be any red-blue sequence. Then $P$ has a 2-page bichromatic book-embedding that is consistent with $\sigma$.

Proof. Let $V_{b}$ and $V_{r}$ be the set of blue and red vertices of $P$ respectively, and let $\sigma$ be any red-blue sequence such that $n_{b}=\left|V_{b}\right|$ and $n_{r}=\left|V_{r}\right|$, where $n_{b}$ and $n_{r}$ are the number of blue and red points of $\sigma$, respectively. Denote as $p_{0}, p_{1}, \ldots p_{n-1}$ the points of $\sigma$ in the order they have in $\sigma$. We describe how to construct a 2page bichromatic book embedding of $P$ consistent with $\sigma$. We shall denote with $P_{k}$ the sub-path of $P$ induced by the first $k+1$ vertices of $P$. The $k+1$ vertices of $P_{k}$ are denoted as $v_{0}, v_{1}, \ldots, v_{k}$.

The proof is constructive and adds one vertex and one edge per step to the bichromatic book embedding. At step $k$ all vertices of $P_{k-1}$ have already been added to the bichromatic book embedding, and we add vertex $v_{k}$ and edge $\left(v_{k-1}, v_{k}\right)$. We denote by $\sigma_{k} \subseteq \sigma$ the red-blue sequence consisting of all points representing the vertices of $P_{k}$. We prove by induction that at the end of step $k$ the following invariants hold:

Property 1. Let $p_{i}$ be the rightmost point of $\sigma_{k}$. Denote as $N B_{k}$ the set of all points of $\sigma \backslash \sigma_{k}$ that precede $p_{i}$ in $\sigma$. All points in $N B_{k}$ have the same color and are all accessible from the same page $\pi$. Furthermore, vertex $v_{k}$ is accessible from $\pi$.

Property 2. Let $p_{j}$ be the point of $\sigma_{k}$ representing vertex $v_{k}$, and let $p_{i}$ be the rightmost point of $\sigma_{k}$. Either $i=j$, or if $j \neq i$ then $c\left(p_{i+1}\right) \neq c\left(p_{j}\right)$.

At step $k=0$ we choose the leftmost point $p_{i}$ of $\sigma$ such that $c\left(p_{i}\right)=c\left(v_{0}\right)$. Properties 1 and 2 trivially hold in this case. At step $k>0$ vertex $v_{k}$ and edge $\left(v_{k-1}, v_{k}\right)$ are added according to the following cases, which depend on the position of the point representing $v_{k-1}$ in $\sigma_{k-1}$.

Case 1. $v_{k-1}$ is represented as the rightmost point $p_{i}$ of $\sigma_{k-1}$. There are three sub-cases (see also Figure 1):

Case 1.a. If $c\left(p_{i+1}\right)=c\left(v_{k}\right)$ then map $v_{k}$ to $p_{i+1}$, and arbitrarily assign $\left(v_{k-1}, v_{k}\right)$ to one of the two pages. No crossing is created by adding edge $\left(v_{k-1}, v_{k}\right)$ because $v_{k-1}$ and $v_{k}$ are represented as consecutive points in the sequence. Properties 1 and 2 hold in this case. Namely, $N B_{k}=N B_{k-1}$ because there is no point between $p_{i}$ and $p_{i+1}$. Hence all points in $N B_{k}$ have the same color and are all accessible from a same page $\pi$ by induction. Also, $v_{k}$ is represented as the rightmost point of $\sigma_{k}$, and hence it is accessible from both pages.

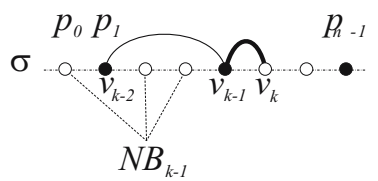

(a)

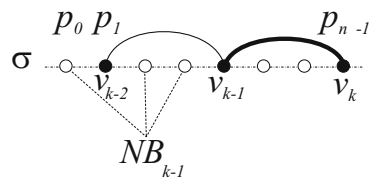

(b)

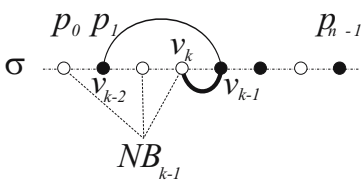

(c)

Fig. 1. Illustrations for Theorem 3 (a) Case 1.a (b) Case 1.b (c) Case 1.c 
It follows that Property 1 holds. Concerning the statement of Property 2, we observe that in this case the point of $\sigma_{k}$ representing vertex $v_{k}$ is the rightmost point.

Case 1.b. Neither $p_{i+1}$ nor the vertices in $N B_{k-1}$ have the same color as $v_{k}$. Map $v_{k}$ to the first vertex $p_{j}$ to the right of $p_{i}$ that has the same color as $v_{k}$, i.e. $j=\min \left\{h \mid h>i \wedge c\left(p_{h}\right)=c\left(v_{k}\right)\right\}$. By induction all points in $N B_{k-1}$ are accessible from a same page $\pi$. We assign edge $\left(v_{k-1}, v_{k}\right)$ to the other page (the one different from $\pi$ ). The addition of edge $\left(v_{k-1}, v_{k}\right)$ does not introduce any crossings, because there is no other edge with an endvertex mapped on a point between $p_{i}$ and $p_{j}$. We have that $N B_{k}=N B_{k-1} \cup\left\{p_{i+1}, p_{i+2}, \ldots, p_{j-1}\right\}$, and that $c\left(p_{i+1}\right)=c\left(p_{i+2}\right)=\cdots=c\left(p_{j-1}\right) \neq c\left(v_{k}\right)$, because $p_{j}$ is the first point after $p_{i}$ such that $c\left(p_{j}\right)=c\left(p_{i}\right)$. It follows that all vertices of $N B_{k}$ have the same color. Also, they are all accessible from $\pi$ because we assign edge $\left(v_{k-1}, v_{k}\right)$ to the page different from $\pi$. Hence the invariant expressed by Property 1 is maintained. Concerning the statement of Property 2, we observe that also in this case the point of $\sigma_{k}$ representing vertex $v_{k}$ is the rightmost point.

Case 1.c. $c\left(p_{i+1}\right) \neq c\left(v_{k}\right), N B_{k-1} \neq \emptyset$, and the vertices of $N B_{k-1}$ have the same color as $v_{k}$. We map $v_{k}$ to the rightmost point $p_{j}$ of $N B_{k-1}$, i.e. $j=\max \left\{h \mid p_{h} \in\right.$ $\left.N B_{k-1}\right\}$. By induction all vertices of $N B_{k-1}$ are accessible from a page $\pi$, and we assign edge $\left(v_{k-1}, v_{k}\right)$ to $\pi$. The addition of edge $\left(v_{k-1}, v_{k}\right)$ does not create a crossing because, by Property $1, v_{k-1}$ and $p_{j}$ are accessible from a common page. We have that $N B_{k}=N B_{k-1} \backslash\left\{p_{j}\right\}$. It follows that the vertices of $N B_{k}$ all have the same color and are all accessible from a page $\pi$ by induction. Point $p_{j}$ is accessible from $\pi$ by induction, and it remains accessible also after that edge $\left(v_{k-1}, v_{k}\right)$ is drawn on $\pi$. Thus Property 1 holds. Property 2 holds since $c\left(p_{i+1}\right) \neq c\left(v_{k}\right)$.

Case 2. $v_{k-1}$ is not represented as the rightmost point $p_{i}$ of $\sigma_{k-1}$. We distinguish three sub-cases (see also Figure 2):

Case 2.a. $c\left(v_{k}\right)=c\left(v_{k-1}\right)$ and $N B_{k-1} \neq \emptyset$. By induction all points of $N B_{k-1}$ have the same color. Also, note that the points of $N B_{k-1}$ plus the point representing $v_{k-1}$ all belong to $N B_{k-2}$ by induction, and hence they all have the same color as $v_{k}$. We map $v_{k}$ to the rightmost point $p_{j}$ of $N B_{k-1}$, i.e. $j=\max \left\{h \mid p_{h} \in\right.$ $\left.N B_{k-1}\right\}$. By induction the vertices of $N B_{k-1}$ are accessible from a page $\pi$; we assign edge $\left(v_{k-1}, v_{k}\right)$ to $\pi$. The addition of $\left(v_{k-1}, v_{k}\right)$ does not create a crossing

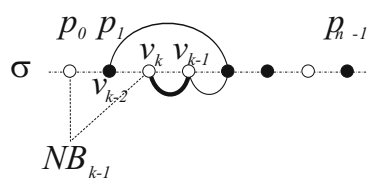

(a)

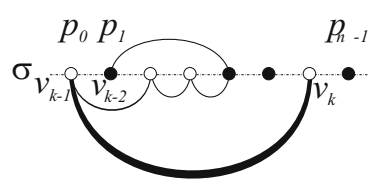

(b)

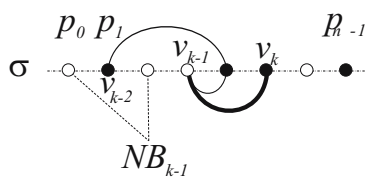

(c)

Fig. 2. Illustrations for Theorem 3 (a) Case 2.a (b) Case 2.b (c) Case 2.c 
because, by Property $1, v_{k-1}$ and $p_{j}$ are accessible from a common page. We have that $N B_{k}=N B_{k-1} \backslash\left\{p_{j}\right\}$, therefore all points of $N B_{k}$ have the same color and are all accessible from page $\pi$ by induction. Point $p_{j}$ was accessible from $\pi$ by induction, and it remains accessible also after edge $\left(v_{k-1}, v_{k}\right)$ is drawn on $\pi$. Thus, Property 1 holds. Property 2 holds because by induction $c\left(p_{i+1}\right) \neq c\left(v_{k-1}\right)$ and $c\left(v_{k}\right)=c\left(v_{k-1}\right)$.

Case 2.b. $c\left(v_{k}\right)=c\left(v_{k-1}\right)$ and $N B_{k-1}=\emptyset$. Choose the first vertex $p_{j}$ to the right of $p_{i}$ such that $p_{j}$ has the same color as $v_{k}$, i.e. $j=\min \left\{h \mid h>i \wedge c\left(p_{h}\right)=c\left(v_{k}\right)\right\}$. Since the point representing $v_{k-1}$ is an element of $N B_{k-2}$, this point is accessible from a page $\pi$ by induction. We assign edge $\left(v_{k-1}, v_{k}\right)$ to $\pi$. Since point $p_{j}$ is to the right of $p_{i}, p_{j}$ is accessible from both pages, and therefore the addition of edge $\left(v_{k-1}, v_{k}\right)$ does not create a crossing. We have that $N B_{k}=p_{i+1}, p_{i+2}, \ldots, p_{j-1}$. Notice that $c\left(p_{i+1}\right)=c\left(p_{i+2}\right)=\cdots=c\left(p_{j-1}\right) \neq c\left(v_{k}\right)$ because $p_{j}$ is the first point after $p_{i}$ such that $c\left(p_{j}\right)=c\left(p_{i}\right)$. It follows that all points of $N B_{k}$ have the same color. Also, they are all accessible from the page different from $\pi$. Vertex $v_{k}$ is accessible from both pages because it is drawn on the rightmost point of $\sigma_{k}$. Therefore the invariants of Property 1 holds. Property 2 trivially holds since $v_{k}$ is represented as the rightmost point of $\sigma_{k}$.

Case 2.c. $c\left(v_{k}\right) \neq c\left(v_{k-1}\right)$. By Property 2 we have that $c\left(p_{i+1}\right)=c\left(v_{k}\right)$. Map $v_{k}$ to $p_{i+1}$. Since the point representing $v_{k-1}$ is an element of $N B_{k-2}$, it is accessible from a page $\pi$. We assign edge $\left(v_{k-1}, v_{k}\right)$ to $\pi$. Since point $p_{i+1}$ is to the right of $p_{i}$, it is accessible from both pages, and therefore the addition of edge $\left(v_{k-1}, v_{k}\right)$ does not create a crossing. We have that $N B_{k}=N B_{k-1}$ because there is no point between $p_{i}$ and $p_{i+1}$. Hence all points in $N B_{k}$ have the same color, and are all accessible from a same page by induction. Also $v_{k}$ is represented as the rightmost point of $\sigma_{k}$ and hence it is accessible from both pages. It follows that both the invariants expressed by Properties 1 and 2 are maintained.

This concludes the proof of this theorem and hence of Theorem 1

\section{References}

1. M. Abellanas, J. Garcia-Lopez, G. Hernández-Peñver, M. Noy, and P. A. Ramos. Bipartite embeddings of trees in the plane. Discrete Applied Mathematics, 93(23):141-148, 1999.

2. J. Akiyama and J. Urrutia. Simple alternating path problem. Discrete Mathematics, 84:101-103, 1990.

3. E. Di Giacomo, W. Didimo, G. Liotta, and S. K. Wismath. Book-embeddability of series-parallel digraphs. Algorithmica. to appear.

4. A. Kaneko, M. Kano, and K. Suzuki. Path coverings of two sets of points in the plane. In J. Pach, editor, Towards a Theory of Geometric Graph, volume 342 of Contempory Mathematics. American Mathematical Society, Providence, 2004.

5. A. Kanenko and M. Kano. Discrete geometry on red and blue points in the plane a survey -. In Discrete and Computational Geometry, volume 25 of Algorithms and Combinatories. Springer, 2003.

6. M. Kaufmann and R. Wiese. Embedding vertices at points: Few bends suffice for planar graphs. Journal of Graph Algorithms and Applications, 6(1):115-129, 2002. 